\title{
Modeling of Photonic Crystal Optical Fibers With Finite Elements
}

\author{
S. Guenneau, A. Nicolet, F. Zolla, and S. Lasquellec
}

\begin{abstract}
The photonic crystal structures have given rise to innovative techniques to build optical fibers. This paper presents finite element magnetic and electric field models for determining the propagation modes in dielectric waveguides. A combination of edge elements for the transverse field and nodal elements for the longitudinal field is used together with a geometric transformation to cope with the open domain. The model is applied to the study of photonic crystal fiber structure. The case of leaky modes is also discussed.
\end{abstract}

Index Terms-Optical fibers, photonic crystals, propagation modes, Whitney finite elements.

\section{INTRODUCTION}

W E CONSIDER a dielectric waveguide of constant cross section $\Omega$ invariant along the $z$ axis and whose permittivity profile $\varepsilon$ is supposed to be a known function (e.g., a piecewise constant function). We are looking for electromagnetic fields $(\mathcal{E}, \mathcal{H})$ solutions of the following Maxwell equations:

$$
\left\{\begin{array}{l}
\operatorname{curl} \mathcal{H}=\varepsilon \frac{\partial \mathcal{E}}{\partial t} \\
\operatorname{curl} \mathcal{E}=-\mu_{0} \frac{\partial \mathcal{H}}{\partial t}
\end{array}\right.
$$

$\mu_{0}$ being the permeability of vacuum.

Furthermore, choosing a time dependence in $e^{-i \omega t}$, and taking into account the invariance of the guide along its $z$ axis, we define time-harmonic two-dimensional (2-D) electric and magnetic fields $\mathbf{E}$ and $\mathbf{H}$ by

$$
\left\{\begin{array}{l}
\mathcal{E}(x, y, z, t)=\Re e\left(\mathbf{E}(x, y) e^{-i(\omega t-\gamma z)}\right) \\
\mathcal{H}(x, y, z, t)=\Re e\left(\mathbf{H}(x, y) e^{-i(\omega t-\gamma z)}\right)
\end{array} .\right.
$$

If $\left[L^{2}\left(\mathbb{R}^{2}\right)\right]^{3}$ denotes the Hilbert space of square integrable functions on $\mathbb{R}^{2}$ with values in $\mathbb{T}^{3}$, we say that $(\mathbf{E}, \mathbf{H})$ is a guided mode when

$$
\left\{\begin{array}{l}
(\gamma, \omega) \in \mathbb{R}_{+}^{2} \\
(\mathbf{E}, \mathbf{H}) \neq(\mathbf{0}, \mathbf{0}) \\
\mathbf{E}, \mathbf{H} \in\left[L^{2}\left(\mathbb{R}^{2}\right)\right]^{3}
\end{array}\right.
$$

where $\omega$ is the angular frequency in the vacuum, and $\gamma$ denotes the propagating constant of the guided mode.

Manuscript received July 4, 2001; revised October 25, 2001

S. Guenneau is with the University of Liverpool, Department of Mathematical Sciences, Liverpool, L69 3BX U.K. (e-mail: guenneau@liverpool.ac.uk).

A. Nicolet, F. Zolla, and S. Lasquellec are with Institut Fresnel, Faculté de Saint Jérôme, F13397 Marseille Cedex 20, France (e-mail andre.nicolet@fresnel.fr).

Publisher Item Identifier S 0018-9464(02)01252-9.
For (E, H) satisfying (1), (2) can be written as

$$
\left\{\begin{array}{l}
\operatorname{curl}_{\gamma} \mathbf{H}=-i \omega \varepsilon_{0} \varepsilon_{r}(x, y) \mathbf{E} \\
\operatorname{curl}_{\gamma} \mathbf{E}=i \omega \mu_{0} \mathbf{H}
\end{array}\right.
$$

where $\varepsilon_{r}$ denotes the relative permittivity (bounded and coercive function) and where $\operatorname{curl}_{\gamma} \mathbf{H}$ and $\operatorname{curl}_{\gamma} \mathbf{E}$ are defined by

$$
\left\{\begin{array}{l}
\operatorname{curl}_{\gamma} \mathbf{H}(x, y)=\operatorname{curl}\left(\mathbf{H}(x, y) e^{i \gamma z}\right) e^{-i \gamma z} \\
\operatorname{curl}_{\gamma} \mathbf{E}(x, y)=\operatorname{curl}\left(\mathbf{E}(x, y) e^{i \gamma z}\right) e^{-i \gamma z} .
\end{array}\right.
$$

Furthermore, $\operatorname{div}_{\gamma}$ being an operator analogously defined as $\operatorname{curl}_{\gamma}$ in (4), it is clear that $\operatorname{div}_{\gamma} \operatorname{curl}_{\gamma} \varphi=0, \forall \varphi \in\left[\mathcal{D}\left(\mathbb{R}^{2}\right)\right]^{3}$, that is, for smooth vector-valued functions $\varphi$. Thus, denoting by $k_{0}$ the wave number $\omega \sqrt{\mu_{0} \varepsilon_{0}}$, we are led to the following system of Maxwell's type:

$$
\left\{\begin{array}{l}
\operatorname{curl}_{\gamma}\left(\varepsilon_{r}^{-1} \operatorname{curl}_{\gamma} \mathbf{H}\right)=k_{0}^{2} \mathbf{H} \\
\operatorname{div}_{\gamma}\left(\mu_{0} \mathbf{H}\right)=0 .
\end{array}\right.
$$

As the geometric domain is unbounded, one of the pitfall in this problem is the presence of a continuous spectrum due to the lack of compacity of the resolvent of the associated operator. It has been proven [2] that the eigenvalues (discrete spectrum) for a given $\gamma$ satisfy the following dispersion relations:

$$
\frac{\gamma^{2}}{\sup _{x \in \mathbb{R}^{2}} \varepsilon_{r}(x)}<\Lambda^{1}(\gamma) \leq \Lambda^{2}(\gamma) \leq \cdots \leq \Lambda^{k}(\gamma) \leq \gamma^{2}
$$

An empty discrete spectrum corresponds to frequencies under the cut-off and an integer $k=1$ to a monomode fiber.

Thanks to the dispersion relations (6), we know that every eigenvalue greater than $\gamma^{2}$ belongs to the essential spectrum, which gives us a numerical criterion to eliminate the modes without any physical meaning.

\section{Finite-Element Modeling: Magnetic Formulation}

The weak form of (5) corresponds to the problem of annulling the following residues [1]:

$$
\begin{aligned}
& \mathcal{R}\left(\gamma ; \mathbf{H}, \mathbf{H}^{\prime}\right)=\int_{\mathbb{R}^{2}} \frac{1}{\varepsilon_{r}} \operatorname{curl}_{\gamma} \mathbf{H} \cdot \overline{\operatorname{curl}_{\gamma} \mathbf{H}^{\prime}} d x d y \\
& \quad+s \int_{\mathbb{R}^{2}} \operatorname{div}_{\gamma} \mathbf{H} \overline{\operatorname{div}_{\gamma} \mathbf{H}^{\prime}} d x d y-k_{0}^{2} \int_{\mathbb{R}^{2}} \mathbf{H} \cdot \overline{\mathbf{H}^{\prime}} d x d y .
\end{aligned}
$$

Here, $\mathbf{H}^{\prime}$ is the weight vector field chosen in the same space as $\mathbf{H}$, the unknown magnetic field. 
The numerical formulation is given by the following residue [2], [3]:

$$
\begin{aligned}
\mathcal{R}(\gamma & \left.\mathbf{H}, \mathbf{H}^{\prime}\right) \\
= & \int_{\Omega} \varepsilon_{r}^{-1}\left(\operatorname{curl}_{t} \mathbf{H}_{t} \cdot \operatorname{curl}_{t} \overline{\mathbf{H}^{\prime}}+\nabla_{t} H_{l} \cdot \nabla_{t} \bar{H}^{\prime}{ }_{l}\right. \\
& \left.-i \gamma \mathbf{H}_{t} \cdot \nabla_{t} \overline{H^{\prime}}{ }_{l}+i \gamma \nabla_{t} H_{l} \cdot \overline{\mathbf{H}^{\prime}}+\gamma^{2} \mathbf{H}_{t} \cdot \overline{\mathbf{H}^{\prime}}\right) d x d y \\
& -k_{0}^{2} \int_{\Omega}\left(\mathbf{H}_{t} \cdot \overline{\mathbf{H}^{\prime}}+H_{l} \overline{H^{\prime}}{ }_{l}\right) d x d y
\end{aligned}
$$

where the unknown field is now a discrete Hilbert space, i.e., with a finite dimension equal to the number of numerical parameters to be determined. This formulation involves both a transverse field $\mathbf{H}_{t}$ in the section of the guide and a longitudinal field $H_{l}$ along its axis. The section of the guide is meshed with triangles and Whitney finite elements [4] are used, i.e., edge elements for the transverse field and node elements for the longitudinal field

$$
\mathbf{H}= \begin{cases}\mathbf{H}_{t}=\sum_{j}^{n} \alpha_{j} \mathbf{w}_{\mathbf{j}}^{\mathbf{e}}(x, y) e^{i \gamma z} & \text { in } \mathbb{R}^{2} \\ H_{l}=\sum_{j}^{n} \beta_{j} w_{j}^{n}(x, y) e^{i \gamma z} & \text { in } \mathbb{R}\end{cases}
$$

where $\alpha_{j}$ denotes the line integral of the transverse component $\mathbf{H}_{t}$ on the edges, and $\beta_{j}$ denotes the line integral of the longitudinal component $H_{z}$ along one unit of length of the axis of the guide (which is equivalent to the nodal value). In addition, $\mathbf{w}_{\mathbf{j}}^{\mathbf{e}}$ and $w_{j}^{n}$ are, respectively, the basis functions of Whitney 1-forms (edge elements) and Whitney o-forms (nodal elements). Moreover, the use of the Whitney elements solves the spurious mode problem in a way similar to the one of the cavities [4].

To see this, it must be noticed that the penalty term involving the divergence is not introduced in the discrete formulation because the use of Whitney elements guarantees the nullity of the divergence in a weak sense. This point will be further developed and discussed in the electric formulation because both cases are completely similar.

To deal with an open problem, a judicious choice of coordinate transformation allows the finite element modeling of the infinite exterior domain [5]. Considering two disks $D(O, A)$ and $D(O, B)$ of center $O=(0,0)$ and radii $A$ and $B>A$ strictly including $\Omega$, we define a corona $C=D(O, B) \backslash \overline{D(O, A)}$. Let $(x, y)$ be a point in $\mathbb{R}^{2} \backslash \overline{D(O, A)}$ (the infinite outer domain) and $(X, Y)$ be a point in $C$, the transformation is then given by

$$
\left\{\begin{array}{l}
x=f_{1}(X, Y)=X[A(B-A)] /[R(B-R)] \\
y=f_{2}(X, Y)=Y[A(B-A)] /[R(B-R)]
\end{array}\right.
$$

where $R$ denotes the Euclidean norm $\sqrt{X^{2}+Y^{2}}$. This transformation may be viewed as a mapping of the finite corona $C$ with the nonorthogonal coordinate system $(X, Y)$ to the infinite domain with the Cartesian coordinate system $(x, y)$.

This way, the finite-element discretization appears as a chained map from the reference space to the transformed space and from the transformed space to the physical space. Using discretizations entirely based on differential forms allows a straightforward formulation of transformation methods by pull-back of the corresponding weighted residuals.

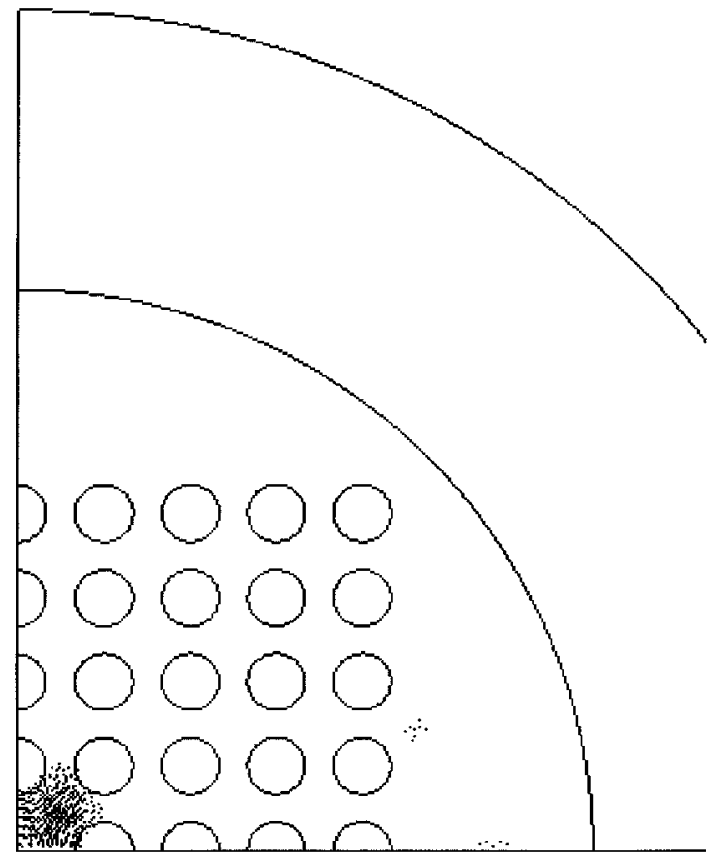

Fig. 1. Localized mode (real part of the transverse magnetic field) in a crystal fiber made of 80 air holes (nearest hole center spacing $\Lambda=3 \mu \mathrm{m}$ ) in a dielectric bulk $(n=2)$ of wave number $k \Lambda=12.446$ and propagating constant $\gamma \Lambda=$ 24

As the eigenvalue problem is linear in $k_{0}^{2}$ and not in $\gamma$, we fix $\gamma \in \mathbb{R}_{+}$and look for $\left(k_{0}^{2}, \mathbf{H}\right)$ satisfying (5). We are thus led to a linear generalized eigenvalue problem that we solve by applying the Lanczos algorithm that is well suited to this kind of large sparse Hermitian matrices [3]. The practical implementation of the model has been performed thanks to the GetDP software [6].

It is also to be noticed that taking Dirichlet boundary conditions at a finite distance (without geometric transformation) from the cross section of the guide allows to consider an operator with a compact resolvent (thus artificially eliminates the continuous spectrum) but it would add the modes of a spurious metallic guide. As for us, we pay a special attention to the lack of compacity of our resolvent operator: the operator remains unbounded under the transformation method and we therefore use the relations (6) as a practical criterion to select the discrete spectrum corresponding to propagating modes and reject the numerical values corresponding to the continuous spectrum.

\section{Application to PhOtONiC CRYSTAL FibERS}

In this work, we study a novel type of optical dielectric waveguide whose properties derive from a periodic arrangement of fibers (not necessarily circular) and from a central structural defect along which the light is guided [7]. In our case, the fiber is an indefinite circular cylinder where air holes are disposed according to a regular pattern (see Fig. 1). The permittivity of the bulk material of the fiber is $4 \varepsilon_{0}$ and the one of the holes is $\varepsilon_{0}$. Fig. 1 shows a localized mode (real component of the transverse magnetic field) in the central defect (no hole). Note that thanks to the symmetry of the problem, only one quarter of the domain is modeled. Fig. 2 shows a closer view to the transverse field in the defect. 


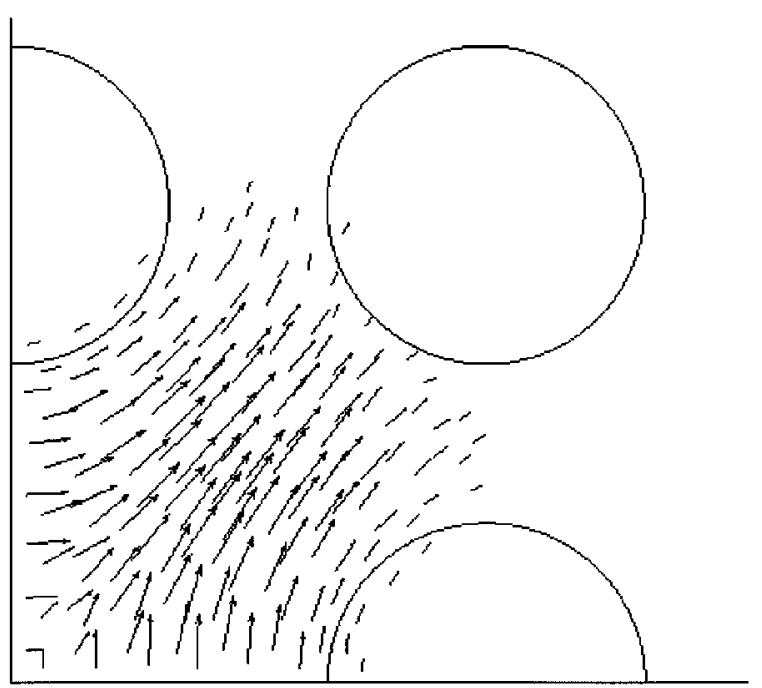

Fig. 2. Zoom on the mode in the core of the fiber (real part of the transverse magnetic field).

\section{LEAKY MODES}

In the previous section, the waveguide is made of holes in a dielectric bulk. The periodic pattern of the holes products the crystal photonic effect responsible of the confinement of the energy in the core of the fiber. This core is in fact a defect corresponding to the absence of a hole in the periodic pattern. It is now interesting to consider the inverted situation where the waveguide is made of dielectric rods disposed in a periodic pattern. There are of course modes propagating in the rods corresponding to the classical waveguide. Nevertheless, what is of interest here are modes propagating in the air to avoid dispersion. The core of the fiber is now a defect corresponding to the absence of rod. It is known [9] that such modes are not really propagating modes in the sense that they correspond to a complex frequency. There is some energy leaking during the propagation of the signal and the imaginary part of the frequency corresponds to a decreasing exponential. The nickname of such modes is "leaky modes." It is not possible to capture such modes with our previous model, but we use the following trick. We do what we have carefully avoided before, which is that the dielectric waveguide is embedded in a cylindrical metallic waveguide. As noted before, working on a bounded domain modifies the structure of the spectrum: there is no more a continuous spectrum but only a discrete spectrum and the $\gamma^{2}$ upper bound in (6) is no more valid and instead

$$
\frac{\gamma^{2}}{\sup _{x \in \mathbb{R}^{2}} \varepsilon_{r}(x)}<\Lambda^{1}(\gamma) \leq \Lambda^{2}(\gamma) \leq \cdots \leq \Lambda^{k}(\gamma) \leq \infty .
$$

What is expected is that the leaky modes with small complex part are now projected on the real axis (see Fig. 3). This approach is somewhat connected to the supercell method.

\section{Finite-ELEMENT MODELING: ELECTRIC FORMULATION}

The presence of metallic wall introduces unknown surface currents and the magnetic formulation is not well suited to take into account such metallic boundary conditions. Therefore, we

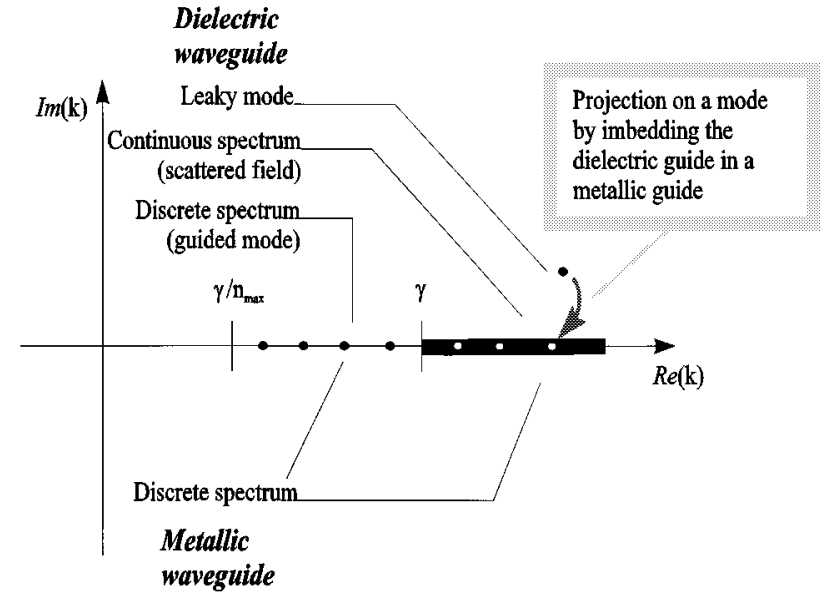

Fig. 3. Leaky modes in dielectric waveguides.

choose now an electric field formulation (dual to the magnetic one) to get simple boundary conditions because the tangential component of the electric field is null [8], what is not the case of the tangential magnetic field equal to the surface current.

The solution of the leaky mode problem is then given by the minimum of the following functional in the weighted Hilbert space $\left[H\left(\mathbb{R}^{2} \backslash \Omega_{m}, d \mathcal{L}\right)\right]^{3}$ where $d \mathcal{L}$ is the Lebesgue measure $\varepsilon_{r} d x d y$ :

$$
\begin{aligned}
& \mathcal{R}\left(\gamma ; \mathbf{E}, \mathbf{E}^{\prime}\right)=\int_{\mathbb{R}^{2} \backslash \Omega_{m}} \operatorname{curl}_{\gamma} \mathbf{E} \cdot \overline{\operatorname{curl}_{\gamma} \mathbf{E}^{\prime}} d \mathcal{L} \\
& \quad+s \int_{\mathbb{R}^{2} \backslash \Omega_{m}} \operatorname{div}_{\gamma} \mathbf{E} \overline{\operatorname{div}_{\gamma} \mathbf{E}^{\prime}} d \mathcal{L}-\mu_{0} \omega^{2} \int_{\mathbb{R}^{2} \backslash \Omega_{m}} \mathbf{E} \cdot \overline{\mathbf{E}^{\prime}} d \mathcal{L} .
\end{aligned}
$$

This problem of minimization admits a unique solution thanks to the penalty term $\int_{\mathbb{R}^{2} \backslash \Omega_{m}} \operatorname{div}_{\gamma} \mathbf{E} \overline{\operatorname{div}_{\gamma} \mathbf{E}^{\prime}} d \mathcal{L}$, which acts in fact as a constraint which forces the nullity of $\operatorname{div}_{\gamma}\left(\varepsilon_{r} \mathbf{E}\right)$ in the exterior of $\Omega_{m}$.

We introduce finite elements in a way similar to the magnetic formulation. Whitney finite elements are used for the electric field, i.e., edge elements for the transverse field and nodal elements for the longitudinal field

$$
\mathbf{E}= \begin{cases}\mathbf{E}_{t}=\sum_{j}^{n} \alpha_{j} \mathbf{w}_{\mathbf{i}}^{\mathbf{e}}(x, y) e^{i \gamma z}, & \text { in } \mathbb{R}^{2} \\ E_{z}=\sum_{j}^{n} \beta_{j} w_{i}^{n}(x, y) e^{i \gamma z}, & \text { in } \mathbb{R}\end{cases}
$$

where $\alpha_{j}$ denotes the line integral of the transverse component $\mathbf{E}_{t}$ on the edges, and $\beta_{j}$ denotes the line integral of the longitudinal component $E_{z}$ along one unit of length of the axis of the guide (what is equivalent to the nodal value). In addition, $\mathbf{w}_{\mathbf{i}}^{\mathbf{e}}$ and $w_{i}^{n}$ are still, respectively, the basis functions of Whitney 1-forms and Whitney 0-forms.

We develop $\operatorname{curl}_{\gamma}$ and $\operatorname{div}_{\gamma}$ in their transverse and longitudinal components

$$
\begin{aligned}
\operatorname{div}_{\gamma} \mathbf{E} & =\operatorname{div}_{t} \mathbf{E}_{t}+i \gamma E_{z} \\
\operatorname{curl}_{\gamma} \mathbf{E} & =\operatorname{curl}_{t} \mathbf{E}_{t} \mathbf{e}_{\mathbf{z}}+\left(\nabla_{t} E_{z}-i \gamma \mathbf{E}_{t}\right) \times \mathbf{e}_{\mathbf{z}}
\end{aligned}
$$




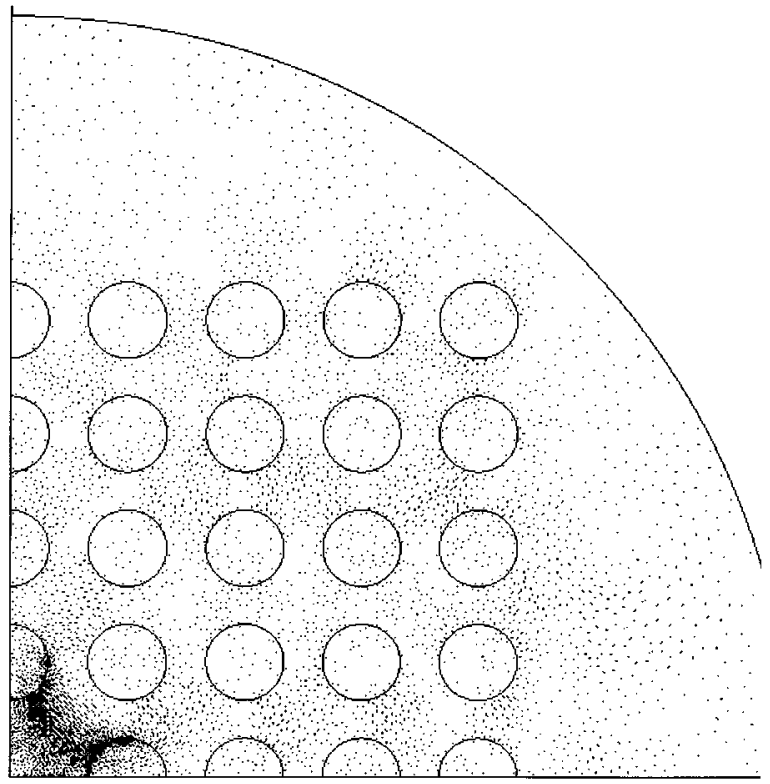

Fig. 4. Localized mode (real part of the transverse electric field) in a crystal fiber made of dielectric rods ( $n=4$ and nearest rod center spacing $\Lambda=3 \mu \mathrm{m}$ ) of wave number $k \Lambda=47.053$ and propagating constant $\gamma \Lambda=21$.

We know that we only have to minimize the following functional:

$$
\begin{aligned}
\mathcal{R}\left(\gamma ; \mathbf{E}, \mathbf{E}^{\prime}\right)= & \int_{\mathbb{R}^{2} \backslash \Omega_{m}}\left(\operatorname{curl}_{t} \mathbf{E}_{t} \cdot \operatorname{curl}_{t} \overline{\mathbf{E}_{t}^{\prime}}-i \gamma \mathbf{E}_{t} \cdot \nabla_{t} \overline{E_{z}^{\prime}}\right. \\
& \left.+i \gamma \nabla_{t} E_{z} \cdot \overline{\mathbf{E}_{t}^{\prime}}+\gamma^{2} \mathbf{E}_{t} \cdot \overline{\mathbf{E}_{t}^{\prime}}\right) d \mathcal{L} \\
& -\mu_{0} \omega^{2} \int_{\mathbb{R}^{2} \backslash \Omega_{m}}\left(\mathbf{E}_{t} \cdot \overline{\mathbf{E}_{t}^{\prime}}+E_{z} \overline{E_{z}^{\prime}}\right) d \mathcal{L}
\end{aligned}
$$

The point here is to notice that the penalty term involving the divergence is not introduced in the discrete formulation because the use of Whitney elements guarantees the nullity of the divergence in a weak sense.

To show this property, one must take

$$
\mathbf{E}^{\prime}=\nabla_{\gamma} \varphi=\nabla_{t} \varphi+i \gamma \varphi \mathbf{e}_{\mathbf{z}}
$$

in (7), where $\varphi$ is a Whitney 0-form (this is allowed since $\nabla_{\gamma} \varphi$ is a Whitney 1-form). The very properties of the Whitney complex ensures that $\operatorname{curl}_{\gamma} \nabla_{\gamma} \varphi$ is null and it follows from (7) that for all $\omega \neq 0$

$$
\int_{\mathbb{R}^{2} \backslash \Omega_{m}} \mathbf{E} \cdot \overline{\nabla_{\gamma} \varphi} d \mathcal{L}=0
$$

which is a weak form of $\operatorname{div}_{\gamma}\left(\varepsilon_{r} \mathbf{E}\right)=0$.

Dispersion relation (6) though no more valid still gives valuable information since the spurious modes have to be searched for frequencies above $\gamma$.

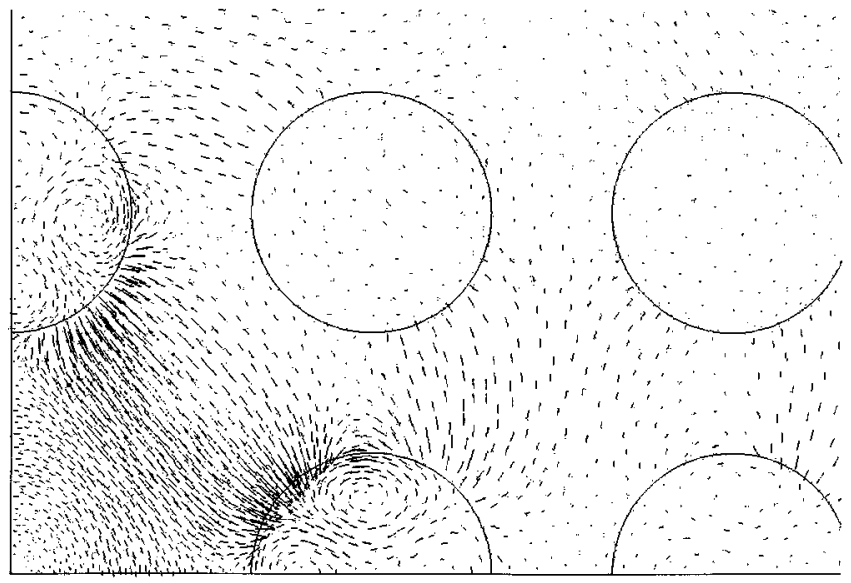

Fig. 5. Zoom on the defect.

Fig. 4 shows a localized mode in a structure made of dielectric rods. The high values of the electric field occur in the air defect in the center of the guide. This is a highly non trivial effect to have the field mainly located in the low index part of the structure. Fig. 5 shows a zoom on the defect.

\section{CONCLUSION}

In this paper, the application of the finite element method to photonic crystal waveguides has been presented. One of the main advantage of this approach with respect to more usual methods in the field is its extreme flexibility with respect to the geometry and the material characteristics of the problem. Reversing the optical index contrast, introducing one or several defects or disturbing the shape or the pattern of the elements of the photonic crystal is not a problem. Future work will concern the improvement of the eigenvalue computation process and the direct search of leaky modes as complex eigenvalues.

\section{REFERENCES}

[1] A.-S. Bonnet, Ph.D. dissertation, Univ. Paris VI, Paris, Fance, 1988.

[2] S. Guenneau, A. Nicolet, F. Zolla, C. Geuzaine, and B. Meys, "A finite element formulation for spectral problems in optical fibers," COMPEL, vol. 20, no. 1, pp. 120-131, 2001.

[3] B. Meys, Ph.D. dissertation, Univ. Liège, Liège, Belgium, 1999.

[4] A. Bossavit, "Solving Maxwell equations in a closed cavity, and the question of spurious modes," IEEE Trans. Magn., vol. 26, pp. 702-705, Mar. 1990.

[5] A. Nicolet, J.-F. Remacle, B. Meys, A. Genon, and W. Legros, "Transformation methods in computational electromagnetism," J. Appl. Phys., vol. 75 , no. 10, 1994.

[6] P. Dular, C. Geuzaine, F. Henrotte, and W. Legros, "A general environment for the treatment of discrete problems and its application to the finite element method," IEEE Trans. Magn., vol. 34, pp. 3395-3398, Nov. 1998.

[7] J. Broeng, D. Mogilevstev, S. E. Barkou, and A. Bjarklev, "Photonic crystal fibers: A new class of optical waveguides," Opt. Fiber Technol., 1999.

[8] Z. Ren, F. Bouillault, A. Razek, A. Bossavit, and J. C. Vérité, "A new hybrid model using electric field formulation for 3-D eddy current problems," IEEE Trans. Magn., vol. 26, pp. 470-473, Mar. 1990.

[9] T. White, "Microstructured optical fibers: A multipole formulation," Res. Project Rep. Univ. Sidney, Sidney, Australia, 2000. 